\title{
Nonlinear dynamics of cilia and flagella
}

\author{
A Hilfinger $\ddagger$, A K Chattopadhyay $\S$ and F Jülicher \\ Max Planck Institute for the Physics of Complex Systems, Nöthnitzer Str. 38, 01187 \\ Dresden, Germany \\ E-mail: julicher@pks.mpg.de
}

\begin{abstract}
Cilia and flagella are hair-like extensions of eukaryotic cells which generate oscillatory beat patterns that can propel micro-organisms and create fluid flows near cellular surfaces. The evolutionary highly conserved core of cilia and flagella consists of a cylindrical arrangement of nine microtubule doublets, called the axoneme. The axoneme is an actively bending structure whose motility results from the action of dynein motor proteins cross-linking microtubule doublets and generating stresses that induce bending deformations. The periodic beat patterns are the result of a mechanical feedback that leads to self-organised bending waves along the axoneme Using a theoretical framework to describe planar beating motion, we derive a nonlinear wave equation that describes the fundamental Fourier mode of the axonemal beat. We study the role of nonlinearities and investigate how the amplitude of oscillations increases in the vicinity of an oscillating instability. We furthermore present numerical solutions of the nonlinear wave equation for different boundary conditions. We find that the nonlinear waves are well approximated by the linearly unstable modes for amplitudes of beat patterns similar to those observed experimentally.
\end{abstract}

$\ddagger$ Current address: Harvard University, Department of Systems Biology, 200 Longwood Ave, Boston, MA 02115, USA

$\S$ Current address: Dipartimento di Fisica "G. Galilei", Università degli Studi di Padova, via F. Marzolo 8, 35131 Padova, Italy 


\section{Introduction}

Cilia and flagella are hair-like appendages of eukaryotic cells exhibiting regular, wavelike oscillations [1]. Their ability to generate regular beat patterns plays an important role in many systems where motion on a cellular level is required [2]. Examples range from the propulsion of single cells, such as the swimming of sperm, to the transport of fluid along ciliated surfaces, such as the flow of mucus in the trachea. Ciliary and flagellar beat patterns are generated by an active structure called the axoneme which consists of nine microtubule doublets arranged in a cylindrical geometry $[1,3,4]$. A large number of dynein motor proteins are arranged between adjacent microtubule doublets and generate internal stresses within the axoneme that induce relative filament sliding and as a consequence axonemal bending $[5,6,7,8,9,10]$.

Axonemal beat patterns have been the subject of several theoretical analyses trying to elucidate the mechanisms underlying the generation of regular beat patterns $[11,12,13,14,15,16,17,18,19,20,21,22,23,24]$. Recent evidence suggests that the interplay of collectively operating motors together with the elastic microtubules constitutes a mechanical feedback that leads to oscillating instabilities [13, 25, 19]. The resulting travelling wave bending patterns can account for the experimentally observed beat patterns in bull sperm [23].

In the present article we extend previous work, in which the linearly unstable modes near an oscillating instability were analysed to discuss the properties of self-organised beating patterns $[19,23]$, by explicitly discussing the effect of nonlinearities on selforganised beat patterns. We present a nonlinear wave equation that describes the fundamental Fourier mode of planar axonemal beat patterns and derive analytically how the nonlinearities determine the amplitude of the beat pattern beyond the bifurcation

point. Furthermore, we present numerical solutions of the nonlinear wave equation subject to three different boundary conditions.

\section{Dynamic equations of motion}

Motivated by the observation that the beat patterns of many sperm are approximately planar we discuss the dynamics of the axoneme in a plane. Such planar beat patterns can be described by an effective, two-dimensional description of the three-dimensional axonemal structure, in which the axoneme is represented by two elastic rods separated by a fixed distance $a$, corresponding to the axonemal diameter of $185 \mathrm{~nm}[19,23]$. These rods are linked by elastic structural elements and by active force generators, corresponding to the dynein motor proteins. To describe the relative motion of the two rods, we introduce the local sliding displacement $\Delta$ and the local shear force density $f$ exerted by passive elastic and active elements, as illustrated in figure 1 . We denote by $\mathbf{r}(s, t)$ the two-dimensional space curve parameterised by its arc length $s$ describing the shape of the centre line of the axoneme of length $L$, at time $t$. As illustrated in figure 


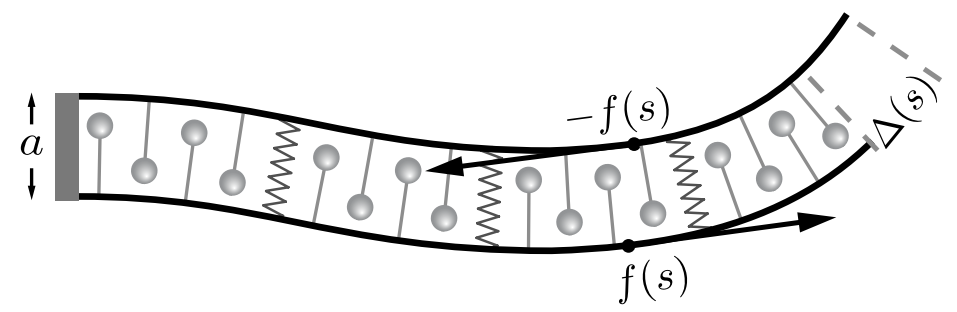

Figure 1. Schematic representation of the effective two-dimensional mechanics of planar beats with two elastic rods sliding relative to each other due to the shear forces generated by active elements. Illustrated are the tangential shear forces $f(s)$ and the local sliding displacement $\Delta(s)$. Elastic structural elements are indicated as springs.

2 , this shape can be characterised by the local tangent angle $\psi(s, t)$ such that

$$
\mathbf{r}(s)=\mathbf{r}(0)+\int_{0}^{s}\left(\cos \psi\left(s^{\prime}\right), \sin \psi\left(s^{\prime}\right)\right) \mathrm{d} s^{\prime},
$$

where we have dropped the explicit time dependence for notational convenience. In this

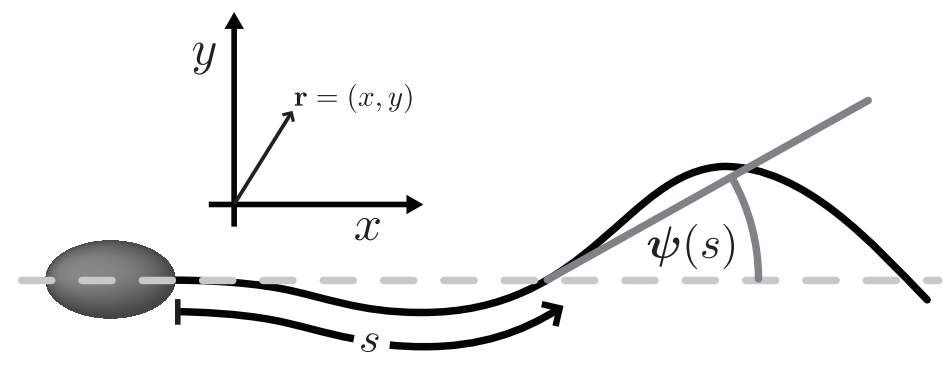

Figure 2. Geometry of the flagellar deformation in the $x, y$-plane. The shape at a given time is described by the local tangent angle $\psi(s)$ as a function of the arc length $s$ along the flagellum.

two-dimensional geometry, the local sliding displacement and the local tangent angle are then related by

$$
\Delta(s)=\Delta_{0}+a(\psi(s)-\psi(0)),
$$

where $\Delta_{0}$ denotes the relative sliding displacement at the base [10, 26, 23]. For simplicity, we ignore hydrodynamic interactions and describe the local hydrodynamic friction by introducing drag coefficients per unit length $\xi_{\|}$and $\xi_{\perp}$ for movements in directions parallel and perpendicular to the axonemal axis, respectively. The dynamics of the axoneme with a bending rigidity $\kappa$ and an internal shear force density $f(s, t)$ is then described by the following set of coupled nonlinear equations [19]

$$
\begin{aligned}
& \partial_{t} \psi=\frac{1}{\xi_{\perp}}\left(-\kappa \psi^{\prime \prime \prime \prime}+a f^{\prime \prime}+\tau^{\prime} \psi^{\prime}+\tau \psi^{\prime \prime}\right)+\frac{1}{\xi_{\|}}\left(\kappa\left(\psi^{\prime}\right)^{2} \psi^{\prime \prime}-a f\left(\psi^{\prime}\right)^{2}+\tau^{\prime} \psi^{\prime}\right) \\
& \tau^{\prime \prime}-\frac{\xi_{\|}}{\xi_{\perp}}\left(\psi^{\prime}\right)^{2} \tau=a\left(f^{\prime} \psi^{\prime}+f \psi^{\prime \prime}\right)-\kappa\left(\left(\psi^{\prime \prime}\right)^{2}+\psi^{\prime} \psi^{\prime \prime \prime}\right)+\frac{\xi_{\|}}{\xi_{\perp}}\left(a f^{\prime} \psi^{\prime}-\kappa \psi^{\prime} \psi^{\prime \prime \prime}\right)
\end{aligned}
$$


where the primes denote derivatives with respect to the arc length $s$, i.e. $\psi^{\prime} \equiv \partial_{s} \psi$. The lateral tension $\tau(s, t)$ ensures that the filament satisfies the local inextensibility constraint $\left(\mathbf{r}^{\prime}\right)^{2}=1$. Note that these equations can be derived from a full threedimensional dynamic description of the axonemal cylinder, restricted to deformations in a plane and do not require the introduction of the effective, two-dimensional axoneme shown in figure 1. [27, 24].

\section{Boundary conditions}

The dynamic equations (3)-(4) are complemented by boundary conditions. While the distal end $(s=L)$ is typically free to move without external constraints, the basal end $(s=0)$ is subjected to external forces and torques. Furthermore, an important aspect of basal dynamics are the mechanical properties of the basal connection which determine the relative sliding between microtubules at the base. Recently, it has been shown that such basal sliding can have an important effect on the shape of the flagellar beat [23]. Following this previous work we characterise the visco-elastic coupling between microtubule doublets at the basal end by a basal elasticity $k_{s}$ and a basal friction $\gamma_{s}$. The basal sliding displacement $\Delta_{0}(t)$ then obeys [23]

$$
\gamma_{s} \partial_{t} \Delta_{0}=-k_{s} \Delta_{0}-\int_{0}^{L} f(s) \mathrm{d} s
$$

and in the limit for large $k_{s}$ and $\gamma_{s}$ basal sliding is suppressed.

The boundary conditions for the tangent angle $\psi(s, t)$ and the tension $\tau(s, t)$ are determined by matching the internal and external torques and forces at the ends [19]. Different experimental conditions imply different boundary conditions at the basal end $s=0$. Motivated by experiments in which the centre of the sperm head is held at a fixed position but potentially free to pivot [23], we describe the dynamics of the head angle $\psi(0, t)$ by introducing an angular elastic modulus $k_{\mathrm{p}}$ and an angular friction coefficient $\gamma_{\mathrm{p}}$. In the following we discuss the cases of (i) a clamped head corresponding to the limit of large $k_{\mathrm{p}}, \gamma_{\mathrm{p}}$ with and without allowing for basal sliding and (ii) a pivoting head corresponding to $k_{\mathrm{p}}, \gamma_{\mathrm{p}}=0$ in the absence of basal sliding. The set of general boundary conditions is summarised in table 1.

Table 1. Boundary conditions of sperm with fixed head position and free tail. We consider specifically the clamped head corresponding to the limit of large $k_{\mathrm{p}}$ and the freely pivoting head limit with $k_{\mathrm{p}}=0$ and $\gamma_{\mathrm{p}}=0$.

\begin{tabular}{ll}
\hline At $s=0$ & At $s=L$ \\
\hline$\kappa \psi^{\prime}+a \int_{0}^{L} f(s) \mathrm{d} s-k_{\mathrm{p}} \psi-\gamma_{\mathrm{p}} \partial_{t} \psi=0$ & $\psi^{\prime}=0$ \\
$\kappa \psi^{\prime \prime \prime}-a \dot{f}-\psi^{\prime} \tau=0$ & $\kappa \psi^{\prime \prime}-a f=0$ \\
$\kappa \psi^{\prime} \psi^{\prime \prime}-a f \psi^{\prime}+\tau^{\prime}=0$ & $\tau=0$ \\
\hline
\end{tabular}




\section{Oscillatory dynamics}

\subsection{Fourier representation}

Time periodic beat patterns can be represented by the temporal Fourier modes $\widetilde{\psi}_{n}(s)$ of the tangent angle

$$
\psi(s, t)=\sum_{n=-\infty}^{\infty} \widetilde{\psi}_{n}(s) \mathrm{e}^{\mathrm{i} n \omega t} .
$$

The Fourier modes $\tilde{f}_{n}(s), \widetilde{\Delta}_{n}(s)$ and $\tilde{\tau}_{n}(s)$ of the local shear force density $f(s, t)$, the local sliding displacement $\Delta(s, t)$ and the tension $\tau(s, t)$ are defined correspondingly.

The motor proteins in the axoneme generate time dependent shear forces which induce dynamic sliding displacements $\Delta(s, t)$. The relation between sliding speed and force is a collective property of the motors together with passive elements cross-linking the axoneme. This effective mechanical property of active and passive elements can be represented as a nonlinear relation in terms of the temporal Fourier modes $[25,19]$

$$
\tilde{f}_{1}=\alpha \widetilde{\Delta}_{1}+\beta \widetilde{\Delta}_{1}\left|\widetilde{\Delta}_{1}\right|^{2}+\mathcal{O}\left(\Delta^{5}\right)
$$

The emergence of spontaneous oscillations is related to negative signs of the real and imaginary parts of the linear response function $\alpha$, resulting from the collective properties of many molecular motors coupled to an elastic element $[25,28,19,29,30]$. The collective effects arise from the dependence of transition rates on the state of the system, as for example introduced by a load dependence of the motor detachment rate [19, 23].

\subsection{Nonlinear waves}

The nonlinear dynamics of the axoneme can then be expressed by coupled differential equations for the discrete Fourier modes of the tangent angle and the tension. The experimentally observed beat patterns of sperm are dominated by their fundamental temporal Fourier mode [23], with higher harmonics contributing to less than $5 \%$ of the wave pattern. We thus neglect in the following higher temporal harmonics of $\psi(t)$.

To simplify the notation in the following, we drop the tilde when referring to temporal Fourier amplitudes, defining $\psi(s) \equiv \widetilde{\psi}_{1}(s), \tau_{0} \equiv \tilde{\tau}_{0}(s), \tau_{2} \equiv \tilde{\tau}_{2}(s)$. Furthermore, we introduce dimensionless parameters $\bar{\omega}, \bar{\alpha}, \bar{\beta}$ and $\bar{\Delta}_{0}$ as defined in Appendix A. Taking into account nonlinearities self-consistently up to cubic terms, equations (3),(4) and (7) lead to the following set of coupled nonlinear equations for the dominant modes $\psi, \tau_{0}$ and $\tau_{2}$

$$
\begin{aligned}
\mathrm{i} \bar{\omega} \psi & =-\dddot{\psi}+\bar{\alpha} \ddot{\psi}+\bar{\beta} \partial_{\bar{s}}^{2}\left[\left(\psi+\bar{\Delta}_{0}-\psi(0)\right)\left|\psi+\bar{\Delta}_{0}-\psi(0)\right|^{2}\right]+\partial_{\bar{s}}\left(\tau_{0} \dot{\psi}+\tau_{2} \dot{\psi}^{*}\right) \\
& +\frac{\xi_{\perp}}{\xi \|}\left[\partial_{\bar{s}}\left(|\dot{\psi}|^{2} \dot{\psi}\right)-2 \bar{\alpha}\left(\psi+\bar{\Delta}_{0}-\psi(0)\right)|\dot{\psi}|^{2}-\bar{\alpha}^{*}\left(\psi^{*}+\bar{\Delta}_{0}^{*}-\psi^{*}(0)\right) \dot{\psi}^{2}+\dot{\tau}_{0} \dot{\psi}+\dot{\tau}_{2} \dot{\psi}^{*}\right] \\
\ddot{\tau}_{0}= & 2 \operatorname{Re}\left\{\bar{\alpha} \partial_{\bar{s}}\left[\left(\psi+\bar{\Delta}_{0}-\psi(0)\right) \dot{\psi}^{*}\right]\right\}-\partial_{\bar{s}}^{2}\left(|\dot{\psi}|^{2}\right)+2 \frac{\xi_{\|}}{\xi \perp}\left(|\dot{\psi}|^{2} \operatorname{Re}\{\bar{\alpha}\}-\operatorname{Re}\left\{\dot{\psi}^{*} \dddot{\psi}\right\}\right) \\
\ddot{\tau}_{2}= & \bar{\alpha} \partial_{\bar{s}}\left[\left(\psi+\bar{\Delta}_{0}-\psi(0)\right) \dot{\psi}\right]-\partial_{\bar{s}}(\dot{\psi} \ddot{\psi})+\frac{\xi_{\|}}{\xi \perp}\left(\bar{\alpha} \dot{\psi}^{2}-\ddot{\psi} \dddot{\psi}\right)
\end{aligned}
$$


Here the dots denote derivatives with respect to the rescaled arc length $\bar{s}=s / L$ and complex conjugates are denoted by asterisks. In equation (8) we have also introduced the dimensionless linear contribution to the fundamental Fourier mode of the basal sliding displacement [23]

$$
\bar{\Delta}_{0}=\frac{\bar{\alpha}}{\mathrm{i} \bar{\omega} \bar{\gamma}_{s}+\bar{k}_{s}+\bar{\alpha}}\left(\psi(0)-\int_{0}^{1} \psi(\bar{s}) \mathrm{d} \bar{s}\right) .
$$

The corresponding boundary conditions complementing equation (8) are summarised in Appendix A.

\section{Wave amplitudes}

The above system exhibits an oscillating instability or Hopf bifurcation, at which the modes with which the system becomes unstable are described by a linearised wave equation $[19,23]$. In the oscillatory regime close to the bifurcation, finite amplitude solutions to the full nonlinear wave equation (8) are expected to be similar to the linearly unstable modes. The way the amplitude grows and the the way the shape of the unstable modes changes with increasing distance from the bifurcation are determined by the nonlinear terms of equation (8). In the following we study the effects of nonlinearities near the bifurcation using a systematic expansion.

Linearising the non-linear wave equation (8) in the limit for small amplitudes, the linearly unstable modes denoted by $u_{0}(\bar{s})$ satisfy satisfy the equation $[11,19,23]$

$$
\mathcal{L}_{\mathrm{c}} u_{0}(\bar{s})=0,
$$

subject to appropriate boundary conditions $[19,23]$. where we have defined the linear operator

$$
\mathcal{L}(\bar{\alpha}, \bar{\omega})=\mathrm{i} \bar{\omega}+\partial_{\bar{s}}^{4}-\bar{\alpha} \partial_{\bar{s}}^{2} \quad .
$$

Note that the amplitude of the linear mode $u_{0}$ is not determined by the linear equation (9). For convenience we normalise $u_{0}$ such that $\int_{0}^{1}\left|u_{0}(\bar{s})\right| \mathrm{d} \bar{s}=1$. Equation (9) together with the appropriate boundary conditions $[19,23]$ constitute a boundary value problem. Nontrivial solutions exist only for pairs of critical values of the dimensionless frequency and response coefficient $\left(\bar{\alpha}_{\mathrm{c}}, \bar{\omega}_{\mathrm{c}}\right)[19,27]$. In the following we will denote $\mathcal{L}_{\mathrm{c}}=\mathcal{L}\left(\bar{\alpha}_{\mathrm{c}}, \bar{\omega}_{\mathrm{c}}\right)$, where $\bar{\alpha}_{\mathrm{c}}$ and $\bar{\omega}_{\mathrm{c}}$ are the values of $\bar{\alpha}$ and $\bar{\omega}$ at the bifurcation point. In figure 3 , the line of critical values are indicated by the solid line representing $\bar{\alpha}_{\mathrm{c}}$ as a function of $\bar{\omega}_{\mathrm{c}}$. Note that there exists a discrete spectrum of such critical lines [19].

We can express solutions of the full nonlinear problem for parameters $\bar{\alpha}=\bar{\alpha}_{\mathrm{c}}+\delta \bar{\alpha}$ and $\bar{\omega}=\bar{\omega}_{\mathrm{c}}+\delta \bar{\omega}$ in the vicinity of the bifurcation point by an expansion of the form

$$
\begin{aligned}
& \psi(\bar{s})=\epsilon u_{0}(\bar{s})+\epsilon^{3} u_{1}(\bar{s})+\mathcal{O}\left(\epsilon^{5}\right) \\
& \tau_{0}(\bar{s})=\epsilon^{2} v(\bar{s})+\mathcal{O}\left(\epsilon^{4}\right) \\
& \tau_{2}(\bar{s})=\epsilon^{2} w(\bar{s})+\mathcal{O}\left(\epsilon^{4}\right) .
\end{aligned}
$$


Here $\epsilon$ is a small dimensionless number that characterises the distance from the bifurcation point by

$$
\delta \bar{\alpha}=\rho \mathrm{e}^{\mathrm{i} \theta} \epsilon^{2} \quad \text { and } \quad \delta \bar{\omega}=\mu \epsilon^{2} \quad,
$$

where we have introduced the real coefficients $\rho$ and $\mu$ as well as the phase $\theta$.

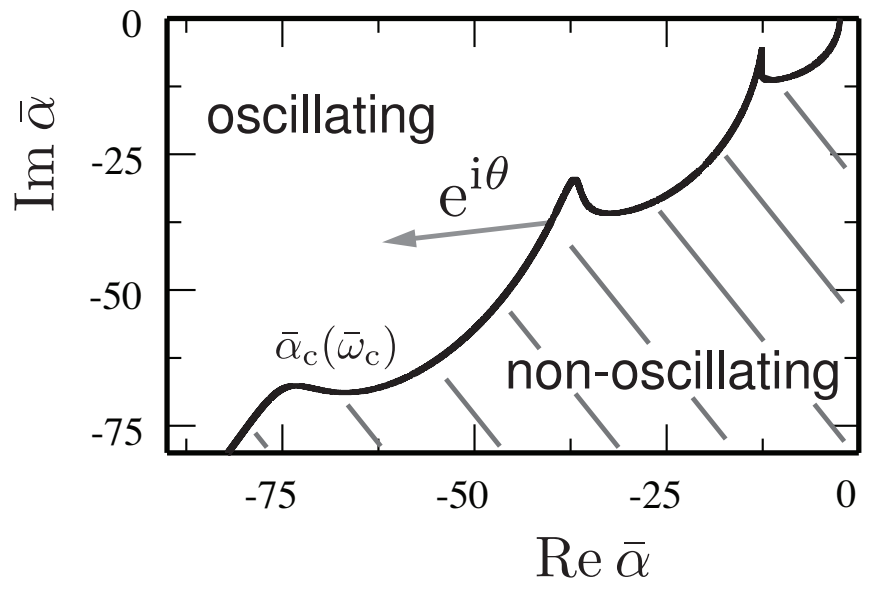

Figure 3. Schematic diagram of the complex plane of motor impedance $\bar{\alpha}_{\mathrm{c}}$. For a given dimensionless frequency $\bar{\omega}_{\mathrm{c}}$, there exists a critical value $\bar{\alpha}_{\mathrm{c}}$ describing an oscillating instability. The line $\alpha_{\mathrm{c}}$ parameterised by $\bar{\omega}_{\mathrm{c}}$ is shown in black. In the dashed region to the right of the line the system is quiescent, whereas to the left of $\bar{\alpha}_{\mathrm{c}}$ it oscillates. The phase theta describes the orientation of a displacement $\delta \bar{\alpha}$ away from a bifurcation point in the complex plane such that $\delta \bar{\alpha}=|\delta \bar{\alpha}| e^{\mathrm{i} \theta}$.

Inserting the ansatz (11) into the wave equation (8), we can solve this equation systematically order by order near a given bifurcation point. First, the linearly unstable mode $u_{0}(\bar{s})$ is determined. Then with $u_{0}(\bar{s})$ known, we can determine the static and dynamic tension profiles $v(\bar{s})$ and $w(\bar{s})$, in terms of $u_{0}(\bar{s})$ as detailed in Appendix B.

Matching terms to third order in $\epsilon$ then leads to an equation for the nonlinear correction $u_{1}(\bar{s})$ to the waveform:

$$
\mathcal{L}_{\mathrm{c}} u_{1}=\rho \mathrm{e}^{\mathrm{i} \theta} \partial_{\bar{s}}^{2} u_{0}-\mathrm{i} \mu u_{0}-\mathcal{N}\left(u_{0}\right),
$$

where the nonlinear terms $\mathcal{N}\left(u_{0}\right)$ are given by

$$
\begin{gathered}
\mathcal{N}\left(u_{0}\right)=\bar{\beta} \partial_{\bar{s}}^{2}\left[\left(u_{0}+\bar{\Delta}_{0}^{(\mathrm{c})}-u_{0}(0)\right)\left|u_{0}+\bar{\Delta}_{0}^{(\mathrm{c})}-u_{0}(0)\right|^{2}\right]+\partial_{\bar{s}}\left(v \dot{u}_{0}+w \dot{u}_{0}^{*}\right) \\
+\frac{\xi_{\perp}}{\xi \|}\left[\partial_{\bar{s}}\left(\left|\dot{u}_{0}\right|^{2} \dot{u}_{0}\right)-2 \bar{\alpha}\left(u_{0}+\bar{\Delta}_{0}^{(\mathrm{c})}-u_{0}(0)\right)\left|\dot{u}_{0}\right|^{2}\right. \\
\left.\quad-\bar{\alpha}^{*}\left(u_{0}^{*}+\bar{\Delta}_{0}^{(\mathrm{c})}-u_{0}^{*}(0)\right)\left(\dot{u}_{0}\right)^{2}+\dot{v} \dot{u}_{0}+\dot{w} \dot{u}_{0}^{*}\right],
\end{gathered}
$$

where $\bar{\Delta}_{0}^{(\mathrm{c})}$ is the basal sliding term evaluated at the bifurcation, as defined by equation (B.3) in the Appendix.

Using equation (13) we can obtain a relation between the coefficients $\rho, \mu$ and $\theta$ without calculating the nonlinear correction $u_{1}$. This is achieved by introducing a 
function $u_{0}^{+}$adjunct to $u_{0}$ which has the property $\mathcal{L}_{\mathrm{c}} u_{0}^{+}=0$ and obeys $\int_{0}^{1} u_{0}^{+} \mathcal{L}_{\mathrm{c}} u=Z$, where $Z$ is a constant and $u$ is an arbitrary function satisfying the boundary conditions, as detailed in Appendix C. Multiplication of (13) with $u_{0}^{+}$and subsequent integration leads to

$$
-\rho \mathrm{e}^{\mathrm{i} \theta} \int_{0}^{1} u_{0}^{+} \partial_{\bar{s}}^{2} u_{0} \mathrm{~d} \bar{s}+\mathrm{i} \mu \int_{0}^{1} u_{0}^{+} u_{0} \mathrm{~d} \bar{s}+\int_{0}^{1} u_{0}^{+} \mathcal{N}\left(\left|u_{0}\right|^{2} u_{0}\right) \mathrm{d} \bar{s}+Z=0 .
$$

We can now discuss the emergence of the unstable mode $\psi(\bar{s})$ and its frequency $\bar{\omega}$ when starting at a bifurcation point at $\bar{\alpha}=\bar{\alpha}_{\mathrm{c}}$ as illustrated in figure 3: moving from $\bar{\alpha}_{\mathrm{c}}$ in a direction given by an angle $\theta$, the above equation (14) describes the beating mode in the oscillatory region of the state diagram characterised by $\bar{\alpha}=\bar{\alpha}_{\mathrm{c}}+|\delta \bar{\alpha}| e^{\mathrm{i} \theta}$. For a chosen value of $\theta$, the values of $\rho$ and $\mu$ can be uniquely determined from the complex equation (14). Equation (12) then describes the increase of the amplitude as

$$
\epsilon=\left(\frac{|\delta \bar{\alpha}|}{\rho}\right)^{1 / 2}
$$

while the frequency changes at the same time by

$$
\delta \bar{\omega}=\frac{\mu}{\rho}|\delta \bar{\alpha}| .
$$

Note that these behaviors depend on the angle $\theta$ chosen. Two special situations are of interest. There exists in general a specific choice $\bar{\theta}$ such $\mu=0$, i.e. in this case the frequency does not change moving in the corresponding direction away from the bifurcation line. Examples for such lines in the complex plane along which the frequency of the unstable modes remains the same as at the bifurcation point $\bar{\alpha}_{\mathrm{c}}$ are displayed in figure 4. A second special choice $\theta=\theta_{\|}$is the direction tangential to the bifurcation line $\bar{\alpha}_{\mathrm{c}}\left(\bar{\omega}_{\mathrm{c}}\right)$. For this choice, equation (14) becomes singular with $\rho \rightarrow \infty$ and $\rho / \mu=\left|\mathrm{d} \bar{\alpha}_{\mathrm{c}} / \mathrm{d} \bar{\omega}_{\mathrm{c}}\right|$. In this case the amplitude $\epsilon$ remains zero, but the frequency changes as it does generally along the bifurcation line [19]. Note also, that the shape and frequency of the beating mode at a point $\alpha$ do not depend on the reference bifurcation point from which it is reached.

The method discussed permits us to calculate amplitude and frequency of unstable modes close to the bifurcation line. In order to study the influence of nonlinearities on the shapes of beating modes, we make use of the above analytical result to solve the nonlinear equations numerically.

\section{Numerical solutions to the nonlinear wave equations}

Periodic and planar beating patterns are solutions to the nonlinear wave equation (8) together with the boundary conditions given in table A2 which constitute a boundary value problem, that can be solved numerically by a shooting and matching procedure. Note that the wave equation is invariant with respect to the overall phase of $\psi(\bar{s})$. To remove this degeneracy we thus impose a boundary condition for the phase of the solution at $\bar{s}=0$. 
In order to obtain numerical solutions that satisfy the wave equation with given boundary conditions, we first determine an approximate solution $\psi(\bar{s}) \approx \epsilon u_{0}(\bar{s})$ close to a bifurcation point $\bar{\alpha}_{\mathrm{c}}, \bar{\omega}_{\mathrm{c}}$ by the method discussed in the previous section. The value of this solution and its derivatives at $\bar{s}=0$ can be used as "seed" estimates for a shooting and matching procedure to find exact numerical results to the full nonlinear equation (8). This procedure consists of first integrating the coupled differential equations, starting with initial values that are consistent with the boundary conditions at $\bar{s}=0$. These initial values are then varied until the obtained solution satisfies also the boundary conditions at $\bar{s}=1$. Solutions further away from the bifurcation can be effectively found by changing the parameter $\bar{\alpha}$ in small incremental steps, such that the known properties of the solution for the preceding value of $\bar{\alpha}$ can be used as a initial estimates for the shooting and matching procedure to converge to the exact solution corresponding to a new value of $\bar{\alpha}$. We use this method to generate a sequence of numerical solutions choosing steps in a direction for which the frequency $\bar{\omega}$ remains constant. Examples of these solutions to equation (8) are displayed in figure 4 for different boundary conditions starting from different bifurcation points.

Parameter values used in these calculations are $\xi_{\perp}=3.4 \times 10^{-3} \mathrm{~N} \cdot \mathrm{s} \cdot \mathrm{m}^{-2}, \xi_{\|}=$ $\xi_{\perp} / 2, \kappa=1.7 \times 10^{-21} \mathrm{~N} \cdot \mathrm{m}^{2}, L=58.3 \mu \mathrm{m}$ as estimated for bull sperm flagella [23, 31]. The nonlinear response coefficient, which determines the oscillation amplitude, has not been measured experimentally. We chose $\bar{\beta}=42$, such that close to the bifurcation points of interest the oscillatory solutions show increasing amplitudes when moving into the unstable region.

The region of stability of the non-oscillating state is indicated in figure 4(A,C,E). The oscillating instability occurs along the solid black line. The real and imaginary parts of such waves as a function of the dimensionless arc length $\bar{s}$ are displayed in figure $4(\mathrm{~B}, \mathrm{D}, \mathrm{F})$ for distinct values of $\alpha$ along the grey line shown in figure $4(\mathrm{~A}, \mathrm{C}, \mathrm{E})$. As indicated by the insets, the amplitude of the modes $A=\int_{0}^{1}|\psi(\bar{s})| \mathrm{d} \bar{s}$ grows continuously with increasing distance from the instability following equation (15) in the limit of small amplitudes.

In contrast to the amplitude, the shape of the beat patterns changes only weakly as illustrated by figure D1 in the Appendix. Solutions to the linearised equations therefore provide good approximations to the full nonlinear problem in the range of examined parameters.

\section{Conclusions and outlook}

In this article we discussed the dynamics of self-organised planar flagellar beat patterns resulting from the co-operative effect of many dynein motors interacting with the elastic microtubule doublets within the axoneme. The dynamics of this system is described by nonlinear equations for the shape and the lateral tension. In order to discuss timeperiodic states, we introduce temporal Fourier modes and derive a nonlinear wave equation for the fundamental modes describing the periodic beat patterns. Extending 


\section{clamped head with basal sliding}

A

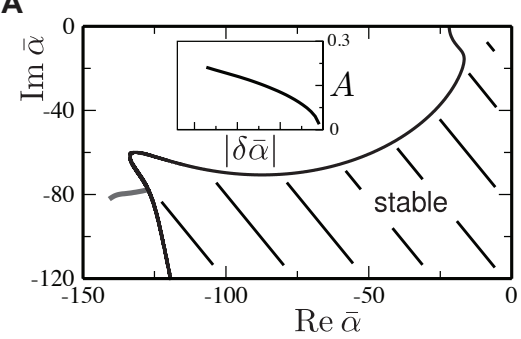

B

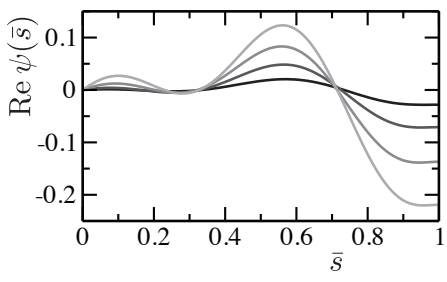

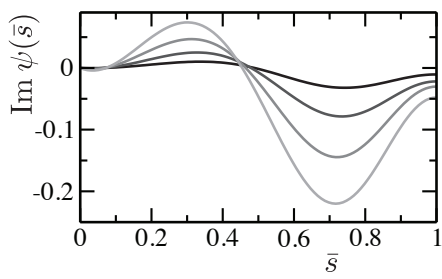

C

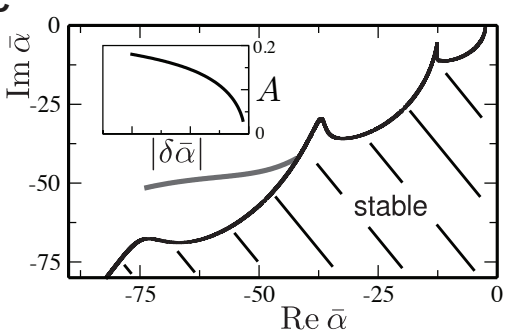

clamped head without basal sliding

\section{D}

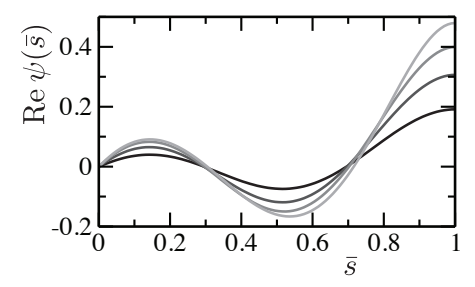

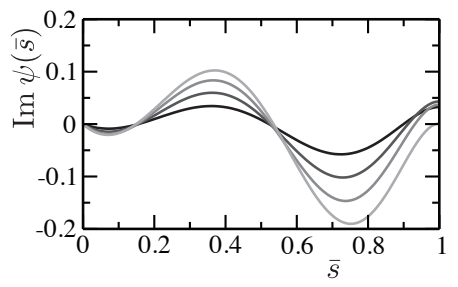

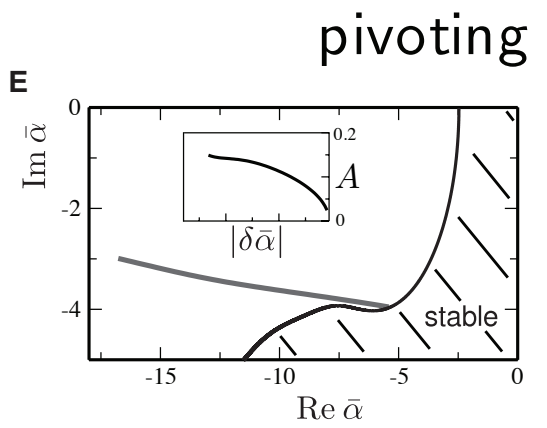

head without basal sliding

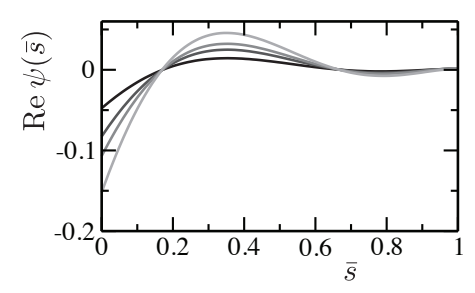

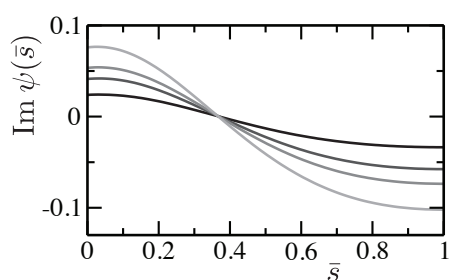

Figure 4. Examples of nonlinear solutions as they grow from the linearly unstable modes. (A) Illustrating how the $26 \mathrm{~Hz}$ solutions of the second branch of unstable modes grow as we go away from the bifurcation in the case of clamped head boundary conditions with basal sliding. The mechanical properties of the basal connection were chosen such that the beat patterns resemble experimentally observed ones, with values for $k_{s}$ and $\gamma_{s}$ as determined in [23]. Indicated is the line along which solutions of equal frequency grow from the bifurcation (grey line) as well as the amplitude $A$ of those these iso-frequent solutions (inset). (B) Real and imaginary part of the fundamental mode of the tangent angle $\psi$ as a function of arc length. Lighter graytone indicates solutions further away from the bifurcation. (C,D) Same as $(A, B)$ but now for the clamped head boundary conditions without basal sliding. Here we look at the bifurcation on the first branch of unstable modes corresponding to a frequency of approximately $28 \mathrm{~Hz}$. (E,F) Same as (C,D) but now showing an example for the freely pivoting head boundary conditions, looking at the bifurcation corresponding to a frequency of approximately $5 \mathrm{~Hz}$. 
previous work, which focussed on the linearly unstable modes near a bifurcation [19, 23], the nonlinear wave equation (8) presented herein describes wave-like patterns of finite amplitude on the oscillating side of the instability. We derived an equation (14) that characterises analytically how nonlinear terms determine the amplitude and frequency of beat patterns in the vicinity of the instability.

Further away from the instability line we analysed the dynamics of cilia and flagella by solving the wave equations numerically using a shooting and matching procedure, extending previous studies on nonlinear beat patterns of passive filaments $[32,33,34]$ to actively beating filaments. We find that the full nonlinear solutions remain qualitatively similar to the waveforms near the instability in the amplitude range discussed herein. Our work thus explains why the linear modes provide a very good approximation to the experimentally observed wave forms of beating sperm [23].

In order to study higher harmonics, as well as transients and interactions between multiple unstable modes, it will be interesting to perform a full numerical simulation of axonemal dynamics in the time-domain. In contrast to the work presented herein, such a simulation requires detailed assumptions to describe the specific microscopic motor dynamics.

For a given motor model, the oscillation frequency emerges from the selforganisation of motors and filaments, with the frequency selection resulting from a subtle condition involving the full frequency dependent impedance of motors. In the future, extensions of our work to include discussions of beat frequencies could allow for further comparisons between our theory and experiments by analysing frequency changes observed for varying flagellar length or varying fluid viscosity.

\section{Acknowledgments}

We thank K. Kruse, A. Vilfan, I. Riedel-Kruse and J. Howard for many helpful discussions. AKC acknowledges the Marie Curie Foundation research fellowship number MIFI-CT-2005-008608 for partial research support.

\section{Appendix A. Dimensionless parameters and boundary conditions}

In the following we define the dimensionless parameters and provide explicit expressions for the boundary conditions that specify the fundamental modes. In order not to

Table A1. Summary of the relations between the physical parameters and the dimensionless quantities.

\begin{tabular}{lllll}
\hline $\bar{\Delta}_{0}=\Delta_{0} / a$ & $\bar{\omega}=\frac{\omega L^{4}}{\kappa} \xi_{\perp}$ & $\bar{\alpha}=\frac{a^{2} L^{2}}{\kappa} \alpha$ & $\bar{\beta}=\frac{a^{4} L^{2}}{\kappa} \beta$ & $\bar{s}=\frac{s}{L}$ \\
$\bar{\tau}_{i}(\bar{s})=\frac{L^{2}}{\kappa} \tau_{i}(s)$ & $\bar{k}_{s}=\frac{a^{2} L}{\kappa} k_{s}$ & $\bar{\gamma}_{s}=\frac{a^{2}}{L^{3} \xi_{\perp}} \gamma_{s}$ & $\bar{k}_{\mathrm{p}}=\frac{L}{\kappa} k_{\mathrm{p}}$ & $\bar{\gamma}_{\mathrm{p}}=\frac{a^{2}}{L^{3} \xi_{\perp}} \gamma_{\mathrm{p}}$ \\
\hline
\end{tabular}

overburden the notation we have dropped the overbars from $\bar{\tau}_{i}$ in equations when the choice of dimensionless or non-dimensionless form is obvious from the context. 
The physical boundary conditions (see table 1) lead to boundary conditions for the fundamental modes $\psi(\bar{s}), \tau_{0}(\bar{s})$ and $\tau_{2}(\bar{s})$ as summarised in table A2, where we have introduced the nonlinear contribution to the dimensionless basal sliding displacement

$$
\bar{\Delta}_{0}^{(\mathrm{nl})}=\bar{\Delta}_{0}-\frac{\bar{\beta}}{\mathrm{i} \bar{\omega} \bar{\gamma}_{s}+\bar{k}_{s}+\bar{\alpha}} \int_{0}^{1}\left|\psi(\bar{s})-\psi(0)+\bar{\Delta}_{0}\right|^{2}\left(\psi(\bar{s})-\psi(0)+\bar{\Delta}_{0}\right) \mathrm{d} \bar{s} .
$$

Table A2. The boundary conditions for the fundamental modes $\psi(\bar{s}), \tau_{0}(\bar{s})$ and $\tau_{2}(\bar{s})$.

$$
\begin{aligned}
& \left(\bar{k}_{\mathrm{p}}+\mathrm{i} \bar{\omega} \bar{\gamma}_{\mathrm{p}}\right) \psi(0)=\dot{\psi}(0)+\bar{\alpha} \int_{0}^{1}\left[\psi(\bar{s})+\bar{\Delta}_{0}-\psi(0)\right] \mathrm{d} \bar{s} \\
& \quad+\bar{\beta} \int_{0}^{1}\left[\left|\psi(\bar{s})+\bar{\Delta}_{0}-\psi(0)\right|^{2}\left(\psi(\bar{s})+\bar{\Delta}_{0}-\psi(0)\right)\right] \mathrm{d} \bar{s} \\
& \dddot{\psi}(0)=\bar{\alpha} \dot{\psi}(0)+\dot{\psi}(0) \tau_{0}(0)+\dot{\psi}^{*}(0) \tau_{2}(0)+\bar{\beta}\left(2\left|\bar{\Delta}_{0}\right|^{2} \dot{\psi}(0)+\left(\bar{\Delta}_{0}\right)^{2} \dot{\psi}^{*}(0)\right) \\
& \dot{\tau}_{0}(0)=-\partial_{\bar{s}}\left(|\dot{\psi}(0)|^{2}\right)+2 \operatorname{Re}\left\{\bar{\alpha} \bar{\Delta}_{0} \dot{\psi}^{*}(0)\right\} \\
& \dot{\tau}_{2}(0)=-\dot{\psi}(0) \ddot{\psi}(0)+\bar{\alpha} \bar{\Delta}_{0} \dot{\psi}(0) \\
& \dot{\psi}(1)=0 \\
& \ddot{\psi}(1)=\bar{\alpha}\left(\psi(1)+\bar{\Delta}_{0}^{(\mathrm{nl})}+\psi(0)\right)+\bar{\beta}\left|\psi(1)+\bar{\Delta}_{0}-\psi(0)\right|^{2}\left(\psi(1)+\bar{\Delta}_{0}-\psi(0)\right) \\
& \tau_{0}(1)=0 \\
& \tau_{2}(1)=0
\end{aligned}
$$

Note that for the limiting cases under consideration in the main text the boundary conditions simplify as follows. In the general clamped head case we have $\psi(0)=0$, in the absence of basal sliding we furthermore have $\bar{\Delta}_{0}, \bar{\Delta}_{0}^{(\mathrm{nl})}=0$, and for a freely pivoting head without basal sliding we can make use of $k_{\mathrm{p}}, \gamma_{\mathrm{p}}=0$ and $\bar{\Delta}_{0}, \bar{\Delta}_{0}^{(\mathrm{nl})}=0$.

\section{Appendix B. Nonlinear perturbation calculationt to second order}

Formally expanding the linear operator as a Taylor series close to the bifurcation point we obtain

$$
\mathcal{L}(\bar{\alpha}, \bar{\omega})=\mathcal{L}_{\mathrm{c}}-\delta \bar{\alpha} \partial_{\bar{s}}^{2}+\mathrm{i} \delta \bar{\omega}
$$

Substituting the ansatz of (11) and (12) into the nonlinear wave equation (8) reproduces to linear order the equation describing the linearly unstable modes (9) supplemented by the appropriate boundary conditions (explicitly described in [27]).

Matching terms to second order in $\epsilon$ then leads to

$$
\begin{aligned}
& \ddot{v}(\bar{s})=2 \operatorname{Re}\left\{\bar{\alpha}_{\mathrm{c}} \partial_{\bar{s}}\left[\left(u_{0}+\bar{\Delta}_{0}^{(\mathrm{c})}-u_{0}(0)\right) \dot{u}_{0}^{*}\right]\right\}-\partial_{\bar{s}}^{2}\left(\left|\dot{u}_{0}\right|^{2}\right)+2 \frac{\xi_{\|}}{\xi_{\perp}}\left(\left|\dot{u}_{0}\right|^{2} \operatorname{Re}\left\{\bar{\alpha}_{\mathrm{c}}\right\}-\operatorname{Re}\left\{\dot{u}_{0}^{*} \dddot{u}_{0}\right\}\right) \\
& \ddot{w}(\bar{s})=\bar{\alpha}_{\mathrm{c}} \partial_{\bar{s}}\left[\left(u_{0}+\bar{\Delta}_{0}^{(\mathrm{c})}-u_{0}(0)\right) \dot{u}_{0}\right]-\partial_{\bar{s}}\left(\dot{u}_{0} \ddot{u}_{0}\right)+\frac{\xi_{\|}}{\xi_{\perp}}\left(\bar{\alpha}_{\mathrm{c}}\left(\dot{u}_{0}\right)^{2}-\dot{u}_{0} \dddot{u}_{0}\right)
\end{aligned}
$$

where

$$
\bar{\Delta}_{0}^{(\mathrm{c})}=-\frac{\bar{\alpha}_{\mathrm{c}}}{\mathrm{i} \bar{\omega}_{\mathrm{c}} \bar{\gamma}+\bar{k}_{s}+\bar{\alpha}_{\mathrm{c}}} \int_{0}^{1} u_{0}(\bar{s}) \mathrm{d} \bar{s}
$$


is the amplitude of basal sliding to linear order. The above system of equations together with the appropriate boundary conditions [27], allow us to obtain $v(\bar{s}), w(\bar{s})$ for given solutions $u_{0}(\bar{s})$ of the linear problem.

\section{Appendix C. Nonlinear perturbation calculation to third order}

Multiplication of (13) with $u_{0}^{+}(\bar{s})$, and subsequent integration leads to an equation in which the only term involving $u_{1}(\bar{s})$ is given by

$$
\begin{aligned}
\int_{0}^{1} u_{0}^{+} \mathcal{L}_{\mathrm{c}} u_{1}= & \int_{0}^{1} u_{0}^{+}\left(\mathrm{i} \bar{\omega}_{\mathrm{c}} u_{1}+\dddot{u}_{1}-\bar{\alpha}_{\mathrm{c}} \ddot{u}_{1}\right) \\
= & \int_{0}^{1} u_{1}\left(\mathrm{i} \omega_{\mathrm{c}} u_{0}^{+}+\dddot{u}_{0}^{+}-\bar{\alpha}_{\mathrm{c}} \ddot{u}_{0}^{+}\right) \\
& +\left[u_{0}^{+} \dddot{u}_{1}-\dot{u}_{0}^{+} \ddot{u}_{1}+\left(\ddot{u}_{0}^{+}-\bar{\alpha}_{\mathrm{c}} u_{0}^{+}\right) \dot{u}_{1}-\left(\dddot{u}_{0}^{+}-\bar{\alpha}_{\mathrm{c}} \dot{u}_{0}^{+}\right) u_{1}\right]_{\bar{s}=0}^{\bar{s}=1} .
\end{aligned}
$$

In order to eliminate $u_{1}(\bar{s})$ from the bulk term in the above expression we require that $\mathcal{L}_{\mathrm{c}} u_{0}^{+}(\bar{s})=0$, implying that $u_{0}^{+}(\bar{s})$ satisfies the same differential equation as the linear modes $u_{0}(\bar{s})$. The boundary conditions for $u_{0}^{+}(\bar{s})$ are then chosen such that terms proportional to $u_{1}$ vanish at the boundaries $\bar{s}=0$ and $\bar{s}=1$. This can be done by matching terms of $\mathcal{O}(\epsilon)-\mathcal{O}\left(\epsilon^{3}\right)$ in order to determine the boundary conditions for $u_{0}(\bar{s}), u_{1}(\bar{s}), v(\bar{s}), w(\bar{s})[27]$ and then in turn $u_{0}^{+}(\bar{s})$, which leads to the results as summarised in the next sections, in which we present the results that lead to boundary conditions specifying the conjugate mode $u_{0}^{+}(\bar{s})$ and the definition of $Z$ in the amplitude equation (14). In the following we present the results for the different physical boundary conditions.

\section{Appendix C.1. Clamped head without basal sliding}

The boundary conditions for the conjugate linear solutions $u_{0}^{+}(\bar{s})$ are given by

$$
\dot{u}_{0}^{+}(0)=0, \quad \ddot{u}_{0}^{+}(0)=0, \quad u_{0}^{+}(1)=0, \quad \dddot{u}_{0}^{+}(1)=0,
$$

which leads to

$$
\int_{0}^{1} u_{0}^{+} \mathcal{L}_{\mathrm{c}} u_{1}=-u_{0}^{+}(0) A-\dot{u}_{0}^{+}(1) B \equiv Z,
$$

where we have introduced

$$
\begin{aligned}
& A=\rho \mathrm{e}^{\mathrm{i} \theta} \dot{u}_{0}(0)+v(0) \dot{u}_{0}(0)+w(0) \dot{u}_{0}^{*}(0) \\
& B=\rho \mathrm{e}^{\mathrm{i} \theta} u_{0}(1)+\bar{\beta}\left|u_{0}(1)\right|^{2} u_{0}(1) .
\end{aligned}
$$

\section{Appendix C.2. Clamped head with basal sliding}

The boundary conditions for the conjugate linear solutions $u_{0}^{+}(\bar{s})$ are given by

$$
\dot{u}_{0}^{+}(0)=0, \quad \ddot{u}_{0}^{+}(0)=0, \quad u_{0}^{+}(1)=\frac{\bar{\alpha}_{\mathrm{c}}^{2}}{\mathrm{i} \bar{\omega}_{\mathrm{c}}} \frac{1}{\mathrm{i} \bar{\omega}_{\mathrm{c}} \bar{\gamma}+\bar{k}+\bar{\alpha}_{\mathrm{c}}} \dot{u}_{0}^{+}(1), \quad \dddot{u}_{0}^{+}(1)=0,
$$


which leads to $Z$ of the same form as (C.2), but with a more complicated expression for $A$ and $B$ which are now given by

$$
\begin{aligned}
& A=\rho \mathrm{e}^{\mathrm{i} \theta} \dot{u}_{0}(0)+\bar{\beta}\left(2\left|\bar{\Delta}_{0}^{(\mathrm{c})}\right|^{2} \dot{u}_{0}(0)+\left(\bar{\Delta}_{0}^{(\mathrm{c})}\right)^{2} \dot{u}_{0}^{*}(0)\right)+v(0) \dot{u}_{0}(0)+w(0) \dot{u}_{0}^{*}(0) \\
& B=\rho \mathrm{e}^{\mathrm{i} \theta}\left(u_{0}(1)+\bar{\Delta}_{0}^{(\mathrm{c})}\right)+\bar{\beta}\left|u_{0}(1)+\bar{\Delta}_{0}^{(\mathrm{c})}\right|^{2} u_{0}(1)-\frac{\mu \bar{\Delta}_{0}^{(\mathrm{c})}}{\bar{\omega}_{\mathrm{c}}} \bar{\alpha}_{\mathrm{c}} \\
& +\frac{\bar{\alpha}_{\mathrm{c}}^{2}}{\mathrm{i} \bar{\omega}_{\mathrm{c}} \bar{\gamma}+\bar{k}+\bar{\alpha}_{\mathrm{c}}} \frac{1}{\mathrm{i} \bar{\omega}_{\mathrm{c}}}\left(\rho \mathrm{e}^{\mathrm{i} \theta} \dot{u}_{0}(0)-A+\int_{0}^{1} \mathcal{N}\left(\left|u_{0}(\bar{s})\right|^{2} u_{0}(\bar{s})\right) \mathrm{d} \bar{s}\right) \\
& +\frac{1}{\mathrm{i} \bar{\omega}_{\mathrm{c}} \bar{\gamma}+\bar{k}+\bar{\alpha}_{\mathrm{c}}}\left(\bar{\Delta}_{0}^{(\mathrm{c})}\left(\rho \mathrm{e}^{\mathrm{i} \theta}\left(\mathrm{i} \bar{\omega}_{\mathrm{c}} \bar{\gamma}+\bar{k}\right)-\mathrm{i} \mu \bar{\gamma} \bar{\alpha}_{\mathrm{c}}\right)-\bar{\beta} \bar{\alpha}_{\mathrm{c}} \int_{0}^{1}\left|u_{0}(\bar{s})+\bar{\Delta}_{0}^{(\mathrm{c})}\right|^{2}\left(u_{0}(\bar{s})+\bar{\Delta}_{0}^{(\mathrm{c})}\right) \mathrm{d} \bar{s}\right) .
\end{aligned}
$$

Appendix C.3. Freely pivoting head without basal sliding

The boundary conditions for the conjugate linear solutions $u_{0}^{+}(\bar{s})$ are given by

$$
\begin{aligned}
& \dot{u}_{0}^{+}(0)=0, \quad \dddot{u}_{0}^{+}(1)=0, \quad u_{0}^{+}(1)+\mathrm{i} \frac{\alpha_{\mathrm{c}}}{\omega_{\mathrm{c}}} \ddot{u}_{0}^{+}(0)=0 \\
& \dddot{u}_{0}^{+}(0)-\alpha_{\mathrm{c}}\left(\dot{u}_{0}^{+}(0)+\ddot{u}_{0}^{+}(0)-\dot{u}_{0}^{+}(1)\right)=0
\end{aligned}
$$

which leads to

$$
\int_{0}^{1} u_{0}^{+} \mathcal{L}_{\mathrm{c}} u_{1}=-A\left(u_{0}^{+}(0)+\mathrm{i} \frac{\alpha_{\mathrm{c}}}{\omega_{\mathrm{c}}} \ddot{u}_{0}^{+}(0)\right)-B \dot{u}_{0}^{+}(1)-C \ddot{u}_{0}^{+}(0) \equiv Z
$$

where we have introduced

$$
\begin{aligned}
A= & \rho \mathrm{e}^{\mathrm{i} \theta} \dot{u}_{0}(0)+v(0) \dot{u}_{0}(0)+w(0)\left(\dot{u}_{0}\right)^{*}(0) \\
B= & \frac{\rho \mathrm{e}^{\mathrm{i} \theta}}{\bar{\alpha}_{\mathrm{c}}} \ddot{u}_{0}(1)+\frac{1}{\left|\bar{\alpha}_{\mathrm{c}}\right|^{2}} \frac{\bar{\beta}}{\bar{\alpha}_{\mathrm{c}}}\left|\ddot{u}_{0}(1)\right|^{2} \ddot{u}_{0}(1) \\
C= & \frac{\rho \mathrm{e}^{\mathrm{i} \theta}}{\bar{\alpha}_{\mathrm{c}}} \dot{u}_{0}(0)-\bar{\beta} \int_{0}^{1}\left[\left|u_{0}(\bar{s})-u_{0}(0)\right|^{2}\left(u_{0}(\bar{s})-u_{0}(0)\right)\right] \mathrm{d} \bar{s} \\
& +\frac{\bar{\alpha}_{\mathrm{c}}}{\mathrm{i} \bar{\omega}_{\mathrm{c}}}\left[\rho \mathrm{e}^{\mathrm{i} \theta} \dot{u}_{0}(0)+\mathrm{i} \mu\left(u_{0}(0)-\frac{1}{\bar{\alpha}_{\mathrm{c}}} \dot{u}_{0}(0)\right)+\int_{0}^{1} \mathcal{N}\left(\left|u_{0}\right|^{2} u_{0}\right) \mathrm{d} \bar{s}\right]
\end{aligned}
$$

\section{Appendix D. Shape changes of the nonlinear solutions}

\section{References}

[1] I. R. Gibbons. Cilia and flagella of eukaryotes. J Cell Biol, 91(3 Pt 2):107s-124s, 1981.

[2] D. Bray. Cell Movements : From Molecules to Motility. Garland, New York, 2nd edition, 2001.

[3] B. A. Afzelius, R. Dallai, S. Lanzavecchia, and P. L. Bellon. Flagellar structure in normal human spermatozoa and in spermatozoa that lack dynein arms. Tissue \& Cell, 27(3):241-247, 1995.

[4] D. Nicastro, J. R. McIntosh, and W. Baumeister. 3d structure of eukaryotic flagella in a quiescent state revealed by cryo-electron tomography. Proc Natl Acad Sci U S A, 102(44):15889-94, 2005.

[5] P. Satir. Studies on cilia: II. Examination of the distal region of the ciliary shaft and the role of the filaments in motility. J Cell Biol, 26(3):805-34, 1965.

[6] I. R. Gibbons and A. J. Rowe. Dynein - a protein with adenosine triphosphatase activity from cilia. Science, 149(3682):424-\&, 1965.

[7] K. E. Summers and I. R. Gibbons. Adenosine triphosphate-induced sliding of tubules in trypsintreated flagella of sea-urchin sperm. Proc Natl Acad Sci U S A, 68(12):3092-\&, 1971. 

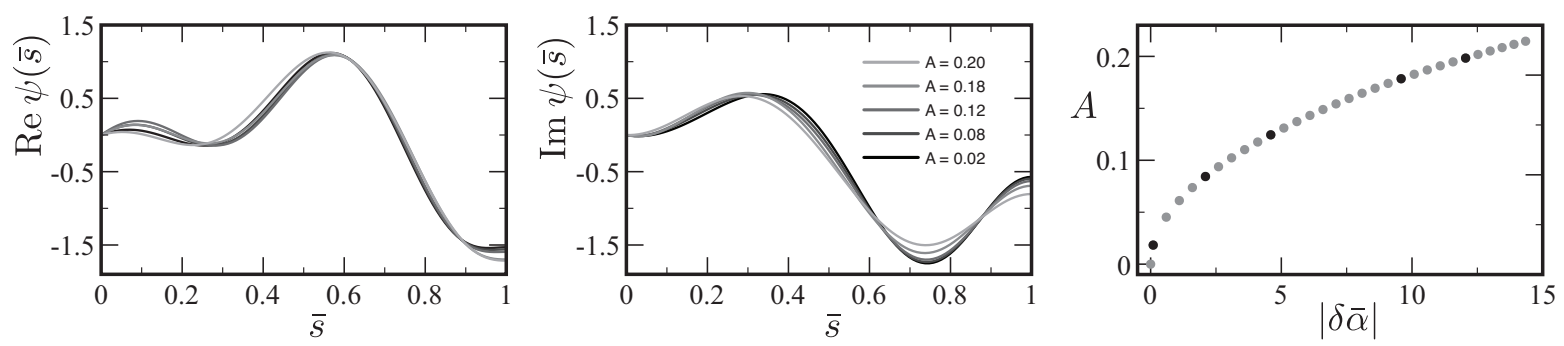

Figure D1. Examples of the shape of the nonlinear solutions as they grow from the linearly unstable modes, corresponding to the $26 \mathrm{~Hz}$ solution of the second branch of unstable modes in the case of clamped head boundary conditions with basal sliding. Shown are the real and imaginary parts of the rescaled nonlinear solutions with the lighter curves corresponding to larger amplitudes. The amplitudes $A$ (in rad) of the selected modes are indicated by black dots in the diagram that shows how the amplitude grows as a function of the distance $|\delta \bar{\alpha}|$ to the bifurcation.

[8] C. J. Brokaw. Direct measurements of sliding between outer doublet microtubules in swimming sperm flagella. Science, 243(4898):1593-6, 1989.

[9] M. E. Porter and W. S. Sale. The $9+2$ axoneme anchors multiple inner arm dyneins and a network of kinases and phosphatases that control motility. J Cell Biol, 151(5):37F-42, 2000.

[10] G. G. Vernon and D. M. Woolley. Microtubule displacements at the tips of living flagella. Cell Motil Cytoskeleton, 52(3):151-60, 2002.

[11] K.E. Machin. Wave propagation along flagella. J Exp Biol, 35(4):796-806, 1958.

[12] C. J. Brokaw. Bend propagation by a sliding filament model for flagella. J Exp Biol, 55(2):289-304, 1971.

[13] C. J. Brokaw. Molecular mechanism for oscillation in flagella and muscle. Proc Natl Acad Sci U $S$ A, 72(8):3102-6, 1975.

[14] C. J. Brokaw and D. R. Rintala. Computer simulation of flagellar movement. III. Models incorporating cross-bridge kinetics. J Mechanochem Cell Motil, 3(2):77-86, 1975.

[15] M. Hines and J.J. Blum. Bend propagation in flagella. II. Incorporation of dynein cross-bridge kinetics into the equations of motion. Biophys J, 25(3):421-41, 1979.

[16] C.B. Lindemann. A geometric clutch hypothesis to explain oscillations of the axoneme of cilia and flagella. J Theor Biol, 168(2):175-89, 1994.

[17] C.B. Lindemann. A model of flagellar and ciliary functioning which uses the forces transverse to the axoneme as the regulator of dynein activation. Cell Motil Cytoskel, 29(2):141-54, 1994.

[18] C.B. Lindemann. Geometric clutch model version 3: The role of the inner and outer arm dyneins in the ciliary beat. Cell Motil Cytoskel, 52(4):242-54, 2002.

[19] S. Camalet and F. Jülicher. Generic aspects of axonemal beating. New Journal of Physics, 2:1-23, 2000 .

[20] C. J. Brokaw. Computer simulation of flagellar movement: VII. conventional but functionally different cross-bridge models for inner and outer arm dyneins can explain the effects of outer arm dynein removal. Cell Motil Cytoskeleton, 42(2):134-48, 1999.

[21] C. J. Brokaw. Computer simulation of flagellar movement VIII: coordination of dynein by local curvature control can generate helical bending waves. Cell Motil Cytoskeleton, 53(2):103-24, 2002.

[22] C. J. Brokaw. Computer simulation of flagellar movement IX. oscillation and symmetry breaking in a model for short flagella and nodal cilia. Cell Motil Cytoskeleton, 60(1):35-47, 2005.

[23] I. Riedel-Kruse, A. Hilfinger, J. Howard, and F. Jülicher. How molecular motors shape the flagellar beat. HFSP Journal, 1(3):192-208, 2007. 
[24] A. Hilfinger and F. Jülicher. The chirality of ciliary beats. Phys. Biol., 5(1):16003, 2008.

[25] F. Julicher and J. Prost. Spontaneous oscillations of collective molecular motors. Phys Rev Lett, 78(23):4510-4513, 1997.

[26] G. G. Vernon and D. M. Woolley. Basal sliding and the mechanics of oscillation in a mammalian sperm flagellum. Biophys J, 87(6):3934-44, 2004.

[27] A. Hilfinger. Dynamics of Cilia and Flagella. Doctoral dissertation, TU Dresden, 2006.

[28] S. Camalet, F. Jülicher, and J. Prost. Self-organized beating and swimming of internally driven filaments. Phys Rev Lett, 82(7):1590-3, 1999.

[29] S.W. Grill, K. Kruse, and F. Jülicher. Theory of mitotic spindle oscillations. Phys Rev Lett, 94(10), 2005.

[30] J. Pecreaux, J.-C. Röper, K. Kruse, F. Jülicher, A. Hyman, S. Grill, and J. Howard. Spindle oscillations during asymmetric cell division require a threshold number of active cortical force generators. Curr Biol, 16:2111-2122, 2006.

[31] J. Howard. Mechanics of Motor Proteins and the Cytoskeleton. Sinauer Associates, Publishers, Sunderland, Mass., 2001.

[32] C. H. Wiggins and R. E. Goldstein. Flexive and propulsive dynamics of elastica at low reynolds number. Phys. Rev. Lett., 80(17):3879-3882, 1998.

[33] C. H. Wiggins, D. Riveline, A. Ott, and R. E. Goldstein. Trapping and wiggling: Elastohydrodynamics of driven microfilaments. Biophys $J, 74(2): 1043-60,1998$.

[34] T. S. Yu, E. Lauga, and A. E. Hosoi. Experimental investigations of elastic tail propulsion at low reynolds number. Physics of Fluids, 18(9), 2006. 